Editorial

\title{
Novel Synthesis and Applications of Metal, Metal Oxides (MOs), and Transition Metal Dichalcogenides (TMDs) for Energy, Sensing, and Memory Applications
}

\author{
Pradip Basnet $\mathbb{D}^{\mathbb{D}},{ }^{1}$ M. Arslan Shehzad, ${ }^{2}$ and $\mathrm{Xi}-\mathrm{Bo} \mathrm{Li}^{3}$ \\ ${ }^{1}$ School of Materials Science \& Engineering, Georgia Institute of Technology, Atlanta, USA \\ ${ }^{2}$ School of Chemical \& Biomolecular Engineering, Georgia Institute of Technology, Atlanta, USA \\ ${ }^{3}$ Research Center of Laser Fusion, China Academy of Engineering Physics, Mianyang, China \\ Correspondence should be addressed to Pradip Basnet; pradip.basnet@mse.gatech.edu \\ Received 26 October 2019; Accepted 26 October 2019; Published 7 November 2019 \\ Copyright (c 2019 Pradip Basnet et al. This is an open access article distributed under the Creative Commons Attribution License, \\ which permits unrestricted use, distribution, and reproduction in any medium, provided the original work is properly cited.
}

Study of metal, metal oxides (MOs), and transition metal dichalcogenides (TMDs) micro-/nanostructures has been an area of interest for researchers recently. These materials exhibit interesting properties for a wide range of applications ranging from electronics to energy conversion. Nanostructures and composites of these metallic nanomaterials due to their high aspect ratio and quantum confinement have been studied for many applications including energy conversion, bio/chemical sensing, and memory applications. Among the different synthesis techniques that are reported for these nanomaterials, physical and chemical methods are the two well-known synthesis routes. In this special issue, we present a selection of invited and contributed articles, which focus on both synthesis and application of these nanomaterials. These accepted manuscripts span a wide range of materials for the applications highlighting novel fabrication techniques as well as suitable test protocols.

In an article entitled "Deposition of Gold Nanoparticles via Galvanic Replacement in DMSO and Their Influence on Formation of Silicon Nanostructures," M. Shepida et al. have reported a lithography-free gold nanoparticle (Au NP) deposition technique on $\mathrm{Si}$ substrates through galvanic replacement (GR) in dimethyl sulfoxide (DMSO) solution. The authors further analyzed the effect of $\mathrm{HAuCl}_{4}$ solution concentration, reaction time, and temperature on the characteristics of the deposited Au NPs. By using the metalassisted chemical etching method, they demonstrated one of the possible applications of synthesized Au NPs, e.g., the formation of nanorods. N. Odaira and Y. Arita in the article entitled "An Estimation of the Thermal Properties of PuRich Metallic Fuel" have studied and reported the estimation of thermal properties of a Pu-rich metallic fuel which has potential to reduce minor actinides with small capacity of fast reactors. Their estimation, using both Nordheim's rule and Wiedemann-Franz law, indicated that this metallic fuel has much lower thermal conductivity and melting point. Furthermore, it was observed that the uranium addition causes increasing thermal conductivity and melting point. In the paper entitled "Synthesis of Mesoporous $\mathrm{TiO}_{2}$ Spheres via the Solvothermal Process and Its Application in the Development of DSSC," S. Velázquez-Martínez et al. have described both the preparation and testing procedure of $\mathrm{TiO}_{2}$ microstructures. They have discussed the usefulness of the solvothermal method for making $\mathrm{TiO}_{2}$ nanoparticles that can be used for solar energy conversion efficiently. For example, the effects of changing solvent-system on the $\mathrm{TiO}_{2}$ products have been studied and reported in detail.

G. Fedorenko et al. in the paper entitled "Oxide Nanomaterials Based on $\mathrm{SnO}_{2}$ for Semiconductor Hydrogen Sensors" reported a synthesis of $\mathrm{SnO}_{2}$ nanostructures which were then characterized for hydrogen sensor applications. Atomically thin $\mathrm{SnO}_{2}(\sim 5.3 \mathrm{~nm})$ was synthesized using the sol-gel method. Additionally, palladium doping was done in order to improve response to hydrogen, and a comparison was made with undoped $\mathrm{SnO}_{2}$. It was observed that doped nanostructures provide fast response and recovery time and a wide measuring range of hydrogen content in air ambient with good repeatability of the sensor signal. Such promising 
properties could make useful sensors based on these nanomaterials for devices intended to determine hydrogen in air. In the paper entitled "Characterization of Layer Number of Two-Dimensional Transition Metal Diselenide Semiconducting Devices Using Si-Peak Analysis," X. Zhang has reported a nondestructive method of characterizing the layer number of "atomically thin" stacking 2D transition metal diselenide nanomaterials, namely, $\mathrm{MoSe}_{2}$ and $\mathrm{WSe}_{2}$, using Raman spectroscopy. The Raman approach for finding the layer number of $\mathrm{MoSe}_{2}$ and $\mathrm{WSe}_{2}$ were reported to be controlled by the change in their optical properties while testing up to four layers.

Concluding the guest editors highlights, we believe that the articles published in Advances in Materials Science and Engineering, special issue, provide useful information as a basis for a better understanding of the metal, MOs, and TMDs micro-/nanostructures and may further aid the scope fabrication and use of multifunctional nanomaterials.

\section{Conflicts of Interest}

The editors declare that they have no conflicts of interest regarding the publication of this Special Issue.

\section{Acknowledgments}

We, guest editors, thank all authors and contributors who submitted their high-quality work for consideration in this special issue. We also appreciate the time and consideration of the reviewers for their valuable feedback in the review process and hence improving the quality of the published articles. We thank one of the guest editors Dr. Sajjad Hussain for his contribution as an editor or deciding on the submitted manuscript. Lastly, we are thankful to Ms. Heba Hamdy for her help.

Pradip Basnet

M. Arslan Shehzad

$\mathrm{Xi}$-Bo $\mathrm{Li}$ 


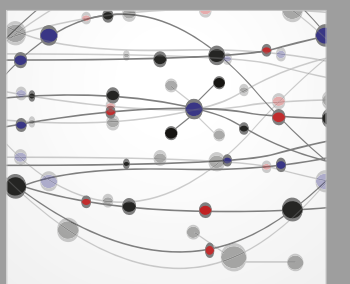

The Scientific World Journal
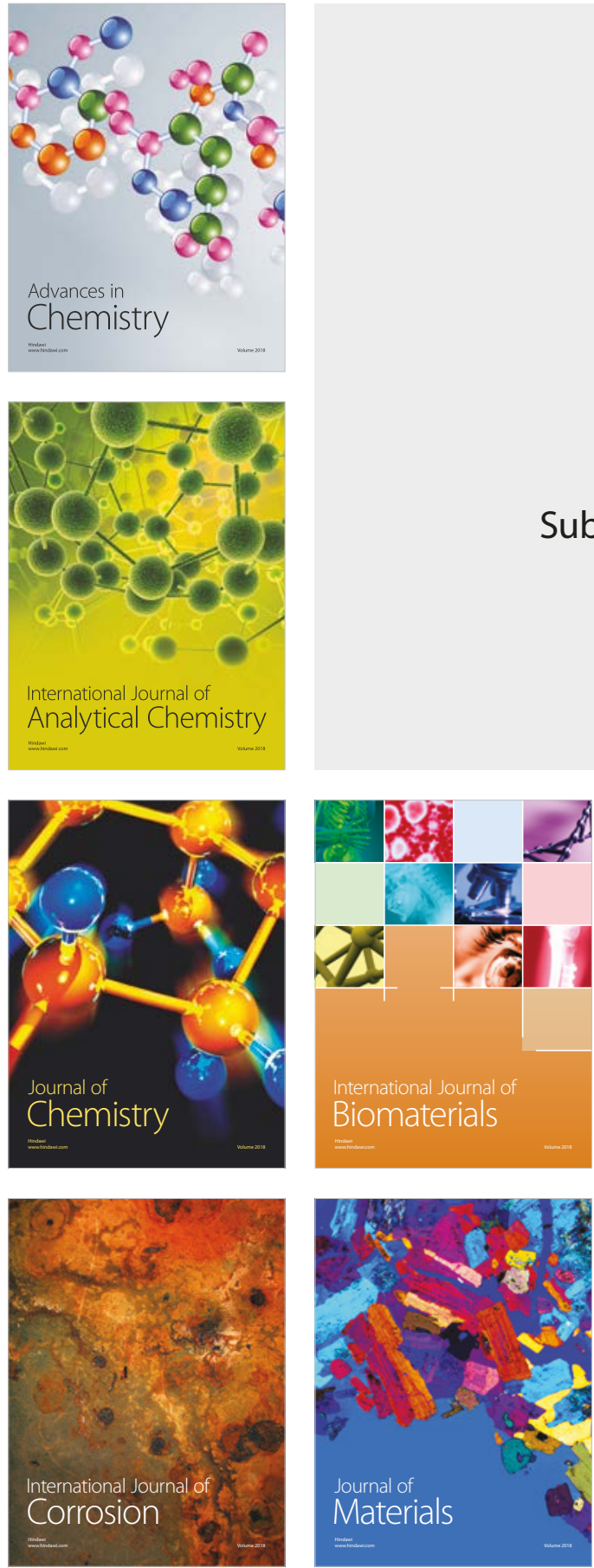

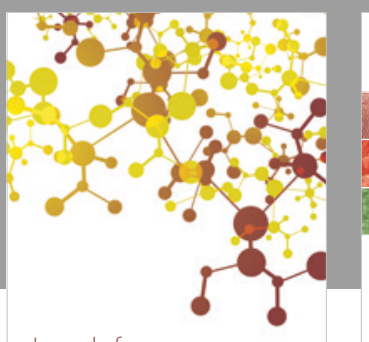

Journal of

Applied Chemistry
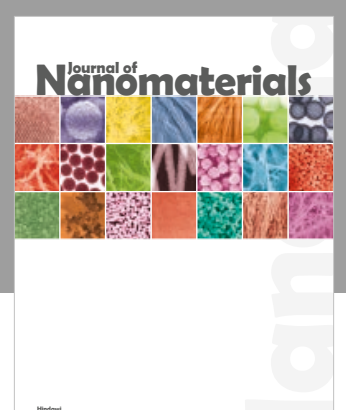

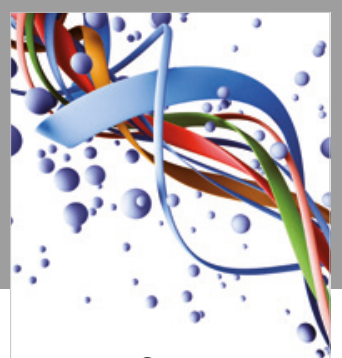

Scientifica

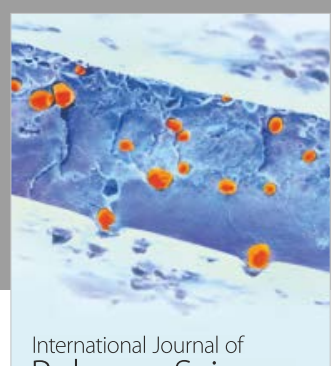

Polymer Science

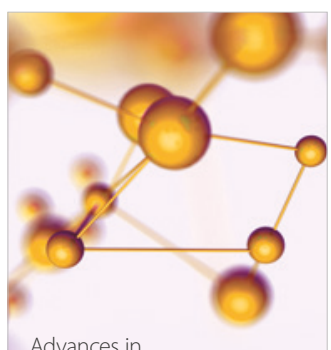

Physical Chemistry
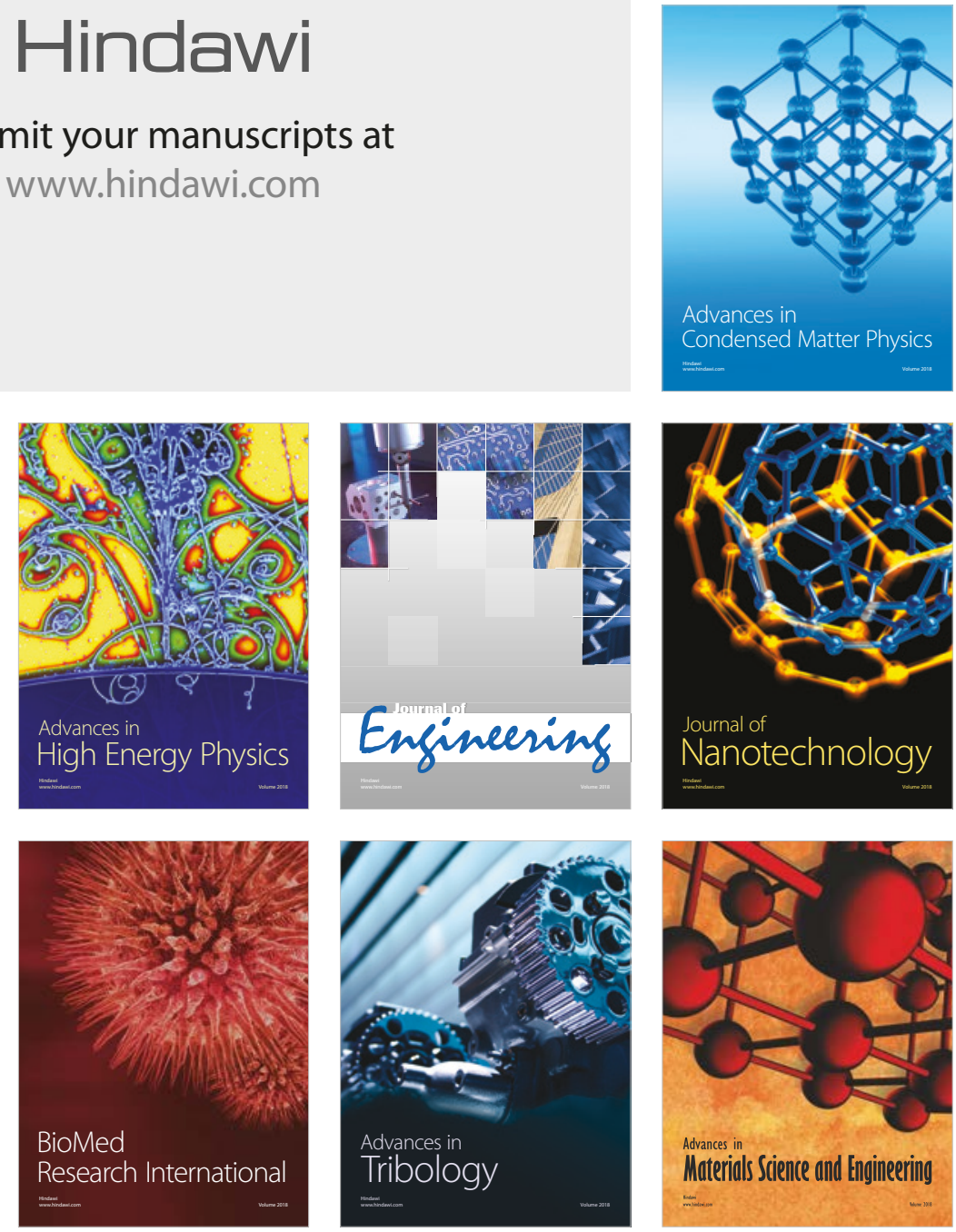